\title{
Multimodality Bayesian Algorithm for Image Reconstruction in Positron Emission Tomography: A Tissue Composition Model
}

\author{
Srikanth Sastry and Richard E. Carson,* Member, IEEE
}

\begin{abstract}
The use of anatomical information to improve the quality of reconstructed images in positron emission tomography (PET) has been extensively studied. A common strategy has been to include spatial smoothing within boundaries defined from the anatomical data. We present an alternative method for the incorporation of anatomical information into PET image reconstruction, in which we use segmented magnetic resonance (MR) images to assign tissue composition to PET image pixels. We model the image as a sum of activities for each tissue type, weighted by the assigned tissue composition. The reconstruction is performed as a maximum a posteriori (MAP) estimation of the activities of each tissue type. Two prior functions, defined for tissue-type activities, are considered. The algorithm is tested in realistic simulations employing a full physical model of the PET scanner.
\end{abstract}

Index Terms - Image reconstruction, maximum a posteriori estimation, positron emission tomography, tissue composition model.

\section{INTRODUCTION}

$\mathbf{T}$ HE expectation maximization (EM) algorithm for maximum likelihood (ML) image reconstruction in positron emission tomography (PET) [1] has been widely studied. While the EM algorithm is flexible in its ability to incorporate the physical and statistical model of projection measurements [2], the algorithm is slow to converge and suffers further from an increase in noise with increasing number of iterations (see, e.g., [3]). Bayesian reconstruction algorithms based on a variety of priors have been studied to extend and regularize the EM-ML method. Initially, priors were used to reduce noise in EM images [4]-[12], by imposing various forms of smoothness constraints. Subsequently, the use of external information to define priors has been explored [13]-[21]. For example, highresolution MR images have been used to define anatomical priors, influencing the formation of boundaries across which the image activity may change abruptly [14]-[17], [19]. Such priors, used in combination with a smoothness constraint,

Manuscript received May 29, 1996; revised April 8, 1997; July 22, 1997. The Associate Editor responsible for coordinating the review of this paper and recommending its publication was R. Leahy. Asterisk indicates corresponding author.

S. Sastry is with the Physical Sciences Laboratory, Division of Computer Research and Technology, Clinical Center, National Institutes of Health, Bethesda, MD 20892 USA.

*R. E. Carson is with the Positron Emission Tomography Department, Clinical Center, National Institutes of Health, Bethesda, MD 20892 USA (email: richard-e-carson@nih.gov.

Publisher Item Identifier S 0278-0062(97)08963-5. model the image being reconstructed as a smooth field within areas delimited by boundaries.

We present an alternative method for using high-resolution MR images in the PET reconstruction process. As a specific example, we consider MR and PET images of the brain. Instead of defining boundaries between regions of different activity levels, we use segmented MR images [22] to assign the "tissue composition" of each pixel in the PET image volume. The activity of a given pixel is modeled as the sum of activities of individual tissue types, weighted by the fraction of each given tissue present in that pixel. The reconstruction algorithm estimates the activities of each tissue type at each image pixel, within a Bayesian framework, wherein we define prior densities for the tissue-type activities. The weighted sum of the individual tissue-type activities is the final reconstructed image. We test this algorithm in simulation studies where we consider a realistic ideal image of a human brain.

The paper is organized as follows: The tissue composition model, upon which our reconstruction method is based, is described in Section II. In Section III, we develop the reconstruction algorithm and the priors to be used. The procedure of the simulation study is described in Section IV, results are given in Section $\mathrm{V}$, and we conclude with a discussion of our results in Section VI.

\section{TISSUE COMPOSITION MODEL}

We first discuss the considerations that motivate the method. In this paper, we consider PET images of the brain only; hence, the discussion makes explicit or implicit reference to these images, even though the considerations here are not restricted to brain images.

Pixel activities in a PET image are strongly dependent on the tissue composition of the pixels. Assume that the object being imaged can be divided into a set of tissue types, e.g., brain tissue can be divided into gray matter (GM), white matter (WM), and cerebrospinal fluid (CSF). The tissue composition of each pixel in the image is then the fraction of the pixel that belongs to each tissue type. Tissue composition can show significant change on a scale comparable to typical pixel sizes. Hence, a realistic idealization of a PET image is that the pixel activities vary significantly across neighboring pixels, and that these activities can to some extent be determined by knowing the tissue composition, if one has good estimates of the activity levels corresponding to different tissue types. This picture 
differs from the idealization where images are viewed as having smoothly varying activities within regions separated by sharp boundaries across which the activities change abruptly.

From this perspective, it is desirable to use anatomical images to determine the tissue composition of pixels rather than, e.g., the location of edges. Hence, the appropriate anatomical prior information for our purposes comes in the form of segmented MR images [22]. Segmentation of an MR image can either be done 1) deterministically, by assigning a tissue type to each MR pixel, or 2) by a fuzzy assignment, i.e., by assigning probabilities for each pixel to be composed of each tissue type (e.g., a given pixel belongs to GM with probability 0.5 , WM with probability 0.4 , etc.). In practice, when the $\mathrm{MR}$ image volume is registered with the PET image volume, more than one MR image pixel is mapped to a given PET pixel. Thus, the resulting "segmentation" in the PET image volume is a specification of the composition of each PET pixel, i.e., what fraction of a PET pixel is composed of GM, etc. The interpretation is clearer for deterministic segmentations than for fuzzy segmentations, though a fuzzy segmentation procedure may in fact be approximating the composition of MR pixels rather than probabilities due to partial volume effects.

By registering segmented MR images with the PET image volume, we assign to each PET pixel a set of (smaller) MR pixels. By averaging the segmentation values (zero or one for deterministic segmentations, or real numbers between zero and one for fuzzy segmentations) of such MR pixels, we evaluate tissue composition (GM, WM, CSF, or other) for each PET pixel. The tissue composition in PET pixel $j$ is given in terms of fractions $p_{j n}$ of each tissue type $n$ ( $n=1$ for CSF, 2 for GM, 3 for WM and 4 for other) with $\sum_{n=1}^{4} p_{j n}=1$. The summation over tissue types $n$ in the rest of the paper has the range $n=1$ to $n=4$.

Next, we discuss how activity at a given PET pixel is modeled in terms of the tissue composition. This model determines the form of the algorithm to be developed below and is thus central to the reconstruction method presented in this paper. In addition to the radionuclide concentration variables $\lambda_{j}$ (at pixel $j$ ) which one considers in a reconstruction procedure, we introduce auxiliary variables $\lambda_{j n}$, where $n$ is the tissue-type index. The variables $\lambda_{j n}$ represent the activity level of tissue type $n$ at pixel $j$, i.e., if pixel $j$ is composed entirely of one tissue type $n\left(p_{j n}=1\right)$, the activity at that pixel would be $\lambda_{j n}$. When pixel $j$ has the composition specified by fractions $p_{j n}$, we model the activity as a sum over activity levels $\lambda_{j n}$, weighted by the fractions $p_{j n}$ of each tissue type. Thus

$$
\lambda_{j}=\sum_{n} p_{j n} \lambda_{j n}
$$

In our study, we use the full physical projection model of the Scanditronix PC2048-15B scanner (details in [2]). The expected value of the projection counts $\overline{Y_{i}}$ of projection ray $i$ is given by

$$
\overline{Y_{i}}=E\left(Y_{i}\right)=\sum_{j} \frac{c_{i j}}{A_{i} N_{i}} \lambda_{j}+R_{i}+S_{i}
$$

where $c_{i j}$ is the geometric probability that pixel $j$ contributes counts to ray $i$ (including detector resolution effects), $A_{i}$ is the attenuation correction factor, $N_{i}$ is the normalization (detector efficiency) correction factor, $R_{i}$ is the estimate of random counts, and $S_{i}$ is the estimate of scatter counts. Pixels were modeled with a "pyramidal" shape, so that activity at any point is determined by bilinear interpolation of the pixel values [2]. With the relation in (1) and $C_{i j n} \equiv c_{i j} p_{j n} / A_{i} N_{i}$, we can write the above expression as

$$
\overline{Y_{i}}=\sum_{j, n} C_{i j n} \lambda_{j n}+R_{i}+S_{i}
$$

Equation (3) expresses the expected projection counts $\overline{Y_{i}}$ in terms of tissue activity levels $\lambda_{j n}$ analogously to (2) above, which expresses $\overline{Y_{i}}$ in terms of the total activity levels $\lambda_{j}$.

In the following Section, we develop a reconstruction method for image activities $\lambda_{j}$, where we first obtain estimates of tissue-type activities $\lambda_{j n}$ iteratively, based on the relation in (3) above. The image activities $\lambda_{j}$ are then obtained using (1). We define prior probability densities for the tissue-type activities $\lambda_{j n}$ and derive an iterative formulation for the maximum a posteriori (MAP) estimates based on the EM algorithm.

\section{THE AlgORITHM}

The a posteriori probability for the tissue-type activities $\left\{\lambda_{j n}\right\}$ (represented below by $\lambda$ ), with measured counts $Y_{i}$ (represented below by $Y$ ) may be written, from Bayes' theorem, as

$$
P(\lambda \mid Y) \propto g(Y \mid \lambda) p(\lambda)
$$

where $g(Y \mid \lambda)$ is the Poisson likelihood function, $p(\lambda)$ is the prior probability density that we define below for the tissuetype activities, and we have omitted the prior density for the counts $Y$.

We consider priors having the form

$$
p(\lambda)=\frac{1}{Z} \exp (-U(\lambda))
$$

where $Z$ is the normalization, and we choose "energy" $U(\lambda)$ to impose appropriate constraints on the reconstructed activities. We have considered two specific prior functions, which we describe after motivating their choice.

The tissue composition model of image activity described in Section II is likely to be most useful in cases where the activity levels for different tissue types are substantially different. This is indeed the case for brain blood flow/metabolism images where GM activities are about a factor of four higher than WM activities, with negligible activity in CSF. If the distribution of activities for any tissue type is narrow in comparison to the overall range of pixel activities, then, a suitable prior for tissue-type activities imposes the condition that at any pixel, the activity for, e.g., GM is "close" to the globalmean GM activity. The first prior we consider imposes this "global constraint" (the "Gaussian prior"). In addition, one may reasonably expect that the variation of activity within each tissue type from pixel to pixel is correlated, i.e., GM 
activity varies smoothly across pixels. This can be modeled as a Markov random field. Corresponding to both these two expectations, the second prior imposes both the global-mean constraint and the spatial smoothness constraint ("SmoothnessGaussian prior").

\section{A. Gaussian Prior}

We express the global-mean constraint via a Gaussian prior probability density $p_{G}(\lambda)$ which is maximum when the tissuetype activities $\lambda_{j n}$ for each tissue type $n$ are equal to the corresponding global-mean activities $\bar{\lambda}_{n}$

$$
p_{G}(\lambda) \propto \exp \left(-U_{G}(\lambda)\right)
$$

with

$$
U_{G}(\lambda)=\sum_{j, n} \frac{\left(\lambda_{j n}-\bar{\lambda}_{n}\right)^{2}}{2 \sigma_{n}^{2}}
$$

The standard deviation (SD) $\sigma_{n}$ controls the width of the Gaussian and, hence, the strength of the constraint imposed by the prior. We choose

$$
\sigma_{n}=\frac{K \bar{\lambda}_{n}}{2(2 \ln 2)^{1 / 2} 100} .
$$

The above choice corresponds to choosing the full width at half maximum (FWHM) of the Gaussian to be $K \%$ of $\bar{\lambda}_{n}$. This choice permits the parameter $K$ to have a physiological interpretation, i.e., the percent variability of activity within GM, WM, etc. We feel that describing the deviation of the pixel activities in a given tissue type in terms of percent variability is natural, since the absolute radioactivity levels can vary significantly from subject to subject depending on radiotracer, injected dose, subject weight, etc. At this stage in the development and testing of the algorithm, we chose a single value of $K$ for all tissue types.

\section{B. Smoothness-Gaussian Prior}

In the second prior, we impose a smoothness constraint in addition to the global-mean constraint. The SmoothnessGaussian probability density prior $p_{S G}(\lambda)$ in this case is

$$
p_{S G}(\lambda) \propto \exp \left(-\left(U_{G}(\lambda)+U_{S}(\lambda)\right)\right)
$$

with

$$
U_{S}(\lambda)=\left(\frac{1}{\gamma}\right) \sum_{j, n} \sum_{k \subset \mathcal{N}_{j}} \frac{\left(\lambda_{j n}-\lambda_{k n}\right)^{2}}{2 \sigma_{j n}^{s 2}}
$$

where $\lambda_{k n}$ are tissue-type activities at pixels $k$ belonging to a neighborhood $\mathcal{N}_{j}$ of pixel $j$, and $\gamma$ is the number of neighbors included in the sum. We choose $\mathcal{N}_{j}$ to include the four pixels surrounding $j$ in the shape of a cross.

As before, $\sigma_{j n}^{s}$ determines the strength of the constraint, but instead of a global value for each tissue type, the SD is defined separately for each pixel based on the local activities. We parametrize $\sigma_{j n}^{s}$ in terms of $K_{S}$ analogously to $\sigma_{n}$ in terms of $K$, with

$$
\sigma_{j n}^{s}=\frac{K_{S} \bar{\lambda}_{j n}}{2(2 \ln 2)^{1 / 2} 100}
$$

where $\bar{\lambda}_{j n}$ is the local mean activity of tissue type $n$ at pixel $j$ evaluated in terms of the neighboring pixel activities.

Note that the inclusion of these priors in the reconstruction does not involve any direct smoothing of the activities $\lambda_{j}$ since the priors apply to tissue-type activities $\lambda_{j n}$ individually. Thus, the resulting images maintain sharp contrast between regions of different tissue type. The parameters $K$ and $K_{S}$ are "control parameters" which determine the strength of the constraints applied and need to be chosen appropriately.

\section{The Iterative Algorithm}

We derive the iteration formula based on the EM algorithm (see e.g., [23]) to find the maximum a posteriori estimates of $\left\{\lambda_{j n}\right\}$. Accordingly, we first define a complete data set $X_{i j n}$ as the number of counts emitted from tissue type $n$ of pixel $j$ detected on projection $i$, such that

$$
Y_{i}=\sum_{j, n} X_{i j n}+R_{i}+S_{i}
$$

Since the $X_{i j n}$ are Poisson distributed, the likelihood for this complete data set is given by

$$
f(X \mid \lambda)=\prod_{i, j, n} \exp \left(-C_{i j n} \lambda_{j n}\right) \frac{\left(C_{i j n} \lambda_{j n}\right)^{X_{i j n}}}{X_{i j n} !}
$$

Given a trial set $\lambda^{(m)}$ of activity values, the conditional expectation of $X_{i j n}$ is

$$
N_{i j n}^{(m)} \equiv E\left(X_{i j n} \mid Y, \lambda^{(m)}\right)=\frac{C_{i j n} \lambda_{j n}^{(m)} Y_{i}}{\sum_{k, l} C_{i k l} \lambda_{k l}^{(m)}+R_{i}+S_{i}}
$$

where $k$ indexes pixels contributing to ray $i$ and $l$ indexes tissue type. Thus, we have the conditional expectation of the log likelihood

$$
\begin{aligned}
& E\left(\ln f(X \mid \lambda) \mid Y, \lambda^{(m)}\right) \\
& \quad=\sum_{i, j, n}-C_{i j n} \lambda_{j n}+N_{i j n}^{(m)} \ln \left(C_{i j n} \lambda_{j n}\right)+R
\end{aligned}
$$

where $R$ is independent of $\lambda$.

In the maximization step, we maximize the a posteriori probability instead of the log likelihood, which is given, in the case of the Gaussian prior, by

$$
E\left(\ln P(X \mid \lambda) \mid Y, \lambda^{(m)}\right)=E\left(\ln f(X \mid \lambda) \mid Y, \lambda^{(m)}\right)-U_{G}(\lambda) .
$$

Analogous to the derivation in [4], we obtain the condition

$$
\frac{\lambda_{j n}^{2}}{\sigma_{n}^{2}}-\lambda_{j n}\left(-\sum_{i} C_{i j n}+\frac{\bar{\lambda}_{n}}{\sigma_{n}^{2}}\right)-\sum_{i} N_{i j n}^{(m)}=0 .
$$

Choosing the positive root of the above quadratic equation results in the following iterative equation for $\lambda$ with the 
Gaussian prior:

$$
\begin{aligned}
\lambda_{j n}^{(m+1)}= & \frac{1}{2}\left(-\sigma_{n}^{2} \sum_{i} C_{i j n}+\bar{\lambda}_{n}\right) \\
& +\frac{1}{2} \sqrt{\left(-\sigma_{n}^{2} \sum_{i} C_{i j n}+\bar{\lambda}_{n}\right)^{2}+4 \sigma_{n}^{2} \sum_{i} N_{i j n}^{(m)}}
\end{aligned}
$$

Note that as $\sigma_{n} \rightarrow 0$ the iterative estimate approaches $\bar{\lambda}_{n}$ [as seen in (18)] while as $\sigma_{n} \rightarrow \infty$, it approaches the conventional EM iteration equation [as seen in (17)]. In the reconstructions discussed, both the expectation and maximization steps are performed simultaneously [i.e., using the same $M$-step values for $\lambda_{j n}$ on the right-hand side of (18)] for all pixels. Examination of (18) shows that the choice of the positive root guarantees positivity of the updated activities [4], once the initial values are chosen to be positive.

The iterative equation for the Smoothness-Gaussian prior, obtained by the same procedure, is

$$
\begin{aligned}
\lambda_{j n}^{(m+1)}= & \frac{1}{2}\left(-S_{j n}^{2} \sum_{i} C_{i j n}+\bar{\Lambda}_{j n}\right) \\
& +\frac{1}{2} \sqrt{\left(-S_{j n}^{2} \sum_{i} C_{i j n}+\bar{\Lambda}_{j n}\right)^{2}+4 S_{j n}^{2} \sum_{i} N_{i j n}^{(m)}}
\end{aligned}
$$

where

$$
\begin{aligned}
S_{j n}^{2} & =\left(\frac{1}{\sigma_{n}^{2}}+\frac{1}{\sigma_{j n}^{s 2}}\right)^{-1} \\
\bar{\Lambda}_{j n} & =\left(\frac{\bar{\lambda}_{n}}{\sigma_{n}^{2}}+\frac{\bar{\lambda}_{j n}}{\sigma_{j n}^{s 2}}\right) S_{j n}^{2}
\end{aligned}
$$

Note that the difference between (18) and (19) lies in the replacement of $\sigma_{n}^{2}$ in (18) by $S_{j n}^{2}$ in (19), and of $\bar{\lambda}_{n}$ by $\bar{\Lambda}_{j n} . S_{j n}^{2}$ and $\bar{\Lambda}_{j n}$ define the constraint strength and mean activity levels calculated by a weighted average of the values specified by the Gaussian and the smoothness constraints individually.

To fully define the priors, the mean activity of each tissue type $\bar{\lambda}_{n}$ must be specified. To specify these values, at the beginning of the reconstruction, we calculated a least-squares estimate of these mean activities from (3) by assuming the activity in each tissue type is uniform, i.e., $\lambda_{j n}=\bar{\lambda}_{n}$ for all pixels $j$. Defining $Y_{i}^{\prime} \equiv Y_{i}-R_{i}-S_{i}$ and $C_{i n}^{\prime} \equiv \Sigma_{j} C_{i j n}$, (3) may be rewritten in matrix form as

$$
Y^{\prime}=C^{\prime} \cdot \bar{\lambda}
$$

where $Y^{\prime}$ is a column vector whose length equals the number of projection rays, $C^{\prime}$ is a rectangular matrix whose number of rows equals the number of projection rays and number of columns equals the number of tissue types, and $\bar{\lambda}$ is a column vector whose length equals the number of tissue types. A least squares estimate of $\bar{\lambda}$ is then obtained in the form

$$
\bar{\lambda}=\left[C^{\prime T} \boldsymbol{C}^{\prime}\right]^{-1} \cdot\left[C^{\prime T} \boldsymbol{Y}^{\prime}\right]
$$

where $\boldsymbol{C}^{\prime T}$ is the transpose of $\boldsymbol{C}^{\prime}$. In this way, no a priori values are required for absolute tissue-type activities.

The reconstruction may then be carried out in one of two ways. First, $\bar{\lambda}_{n}$ (and, therefore, $\sigma_{n}$ ) can be kept fixed at their initial values throughout the iteration process. Alternatively, and for most of the reconstructions presented here, we have evaluated the mean global activities, $\bar{\lambda}_{n}$, iteratively from the current estimates $\lambda^{(m)}$ as

$$
\bar{\lambda}_{n}^{(m)}=\sum_{j} p_{j n} \lambda_{j n}^{(m)} / \sum_{j} p_{j n} .
$$

Similarly, for the Smoothness-Gaussian prior

$$
\bar{\lambda}_{j n}^{(m)}=\frac{1}{\gamma} \sum_{k \subset \mathcal{N}_{j}} \lambda_{k n}^{(m)}
$$

where $\mathcal{N}_{j}$ defines a neighborhood of pixel $j$. In this case, the mean tissue-type activities are initialized to the least-squares estimates. Note that because we update the values of $\bar{\lambda}_{n}$ and $\bar{\lambda}_{j n}$ based on the previous iteration, our implementation is no longer a strictly Bayesian MAP estimation, so convergence may not be guaranteed. In Section VI, we present a comparison of the behavior of the two iterative approaches, i.e., fixed and iteratively updated Bayesian parameters $\bar{\lambda}_{n}$.

\section{SimUlations}

We have tested the algorithm in realistic simulations of the brain based on fluorodyoxyglucose-PET and MR data from a patient with Alzheimer's disease. First we performed a fuzzy segmentation of the MR images using an algorithm based on a material-mixture model of the MR intensity distributions of each tissue [24]. The MR data were then registered to the PET data [25] and the segmentation results were resliced to be coplanar with the PET data. This procedure produced independent images of the probability that each pixel belonged to CSF [Fig. 1(a)], GM [Fig. 1(b)], and WM [Fig. 1(c)]. The pixel size of the (fuzzy) MR segmentations used was $1 \mathrm{~mm}$. Sixteen neighboring pixel values $(4 \times 4)$ in these segmentation images were then averaged to produce the $p_{j n}$ values for the PET pixel size of $4 \mathrm{~mm}$, i.e., the image size was $64 \times 64$ pixels. If the PET pixel size is not a multiple of the MR pixel size, a weighted averaging of segmentation values would be performed. The PET pixel size was chosen to minimize computation time and to allow a direct comparison with our implementation of unconstrained EM-ML [2].

We then assigned mean activities to each tissue type in the ratio of activities GM:WM:CSF:Other $=4: 1: .005: 0$ based on autoradiographic measurements of glucose metabolism [26]. Note that the simulated activities of individual tissue types were not constant across the image. We first generated tissuetype activity values at each pixel as independent Gaussian random numbers distributed around the chosen mean values. The resulting random fields were then spatially smoothed by convolving with a Gaussian kernel with a FWHM of $10 \mathrm{~mm}$. This resulted in a smoothed random field with a distribution of 


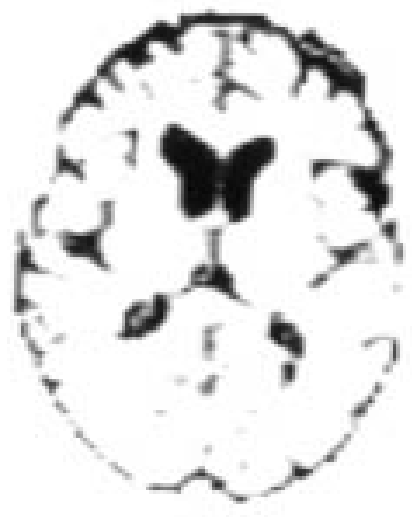

(a)

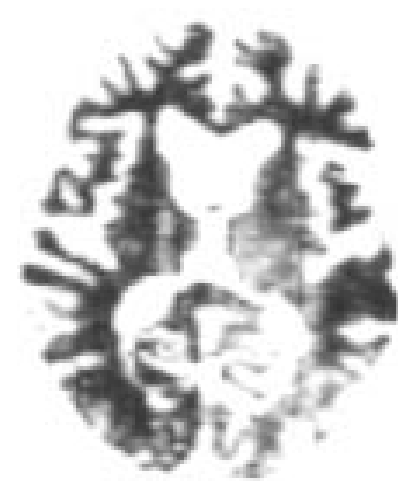

(c)

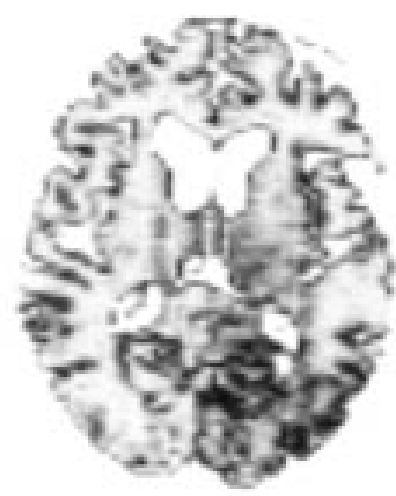

(b)

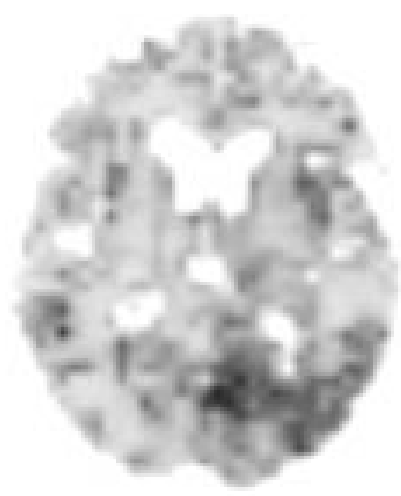

(d)
Fig. 1. Segmented MR images (a) CSF, (b) gray matter, and (c) white matter. (d) "True" PET image created from these segmentations.

values with FWHM $\sim 20 \%$ of their mean activities. The "true" image was then constructed according to (1) and is shown in Fig. 1(d). Histograms of the pixel values are shown in Fig. 2. The outer curves in this figure represent the distribution of simulated values for WM and GM tissue types. The central curve is the histogram of the total pixel activities. Note that the WM and GM histograms lie on either side of the histogram of total activity. This indicates that a majority of pixels have a mixed composition, i.e., there are very few mostly GM or WM pixels. This is due to the original fuzzy segmentation and to the relative sizes of the MR and PET pixels.

We obtained projections from the ideal image by applying the projection model described earlier using the patient data for the attenuation, random, and scatter terms in the model. Details of the physical model (sinogram size, resolution, etc.) are described in [2]. We generated two sets of projections: 1) without Poisson noise, to estimate the root mean squared (rms) bias, and 2) with Poisson noise, to estimate variability of the reconstructed images. Ten independent noisy projection datasets were generated, each with roughly a million counts.

Reconstructions (for 500 iterations) were performed with both the Gaussian and the Smoothness-Gaussian priors, for a range of values of the control parameters $K$ and $K_{S}$. In each case, we evaluated the bias (from noise-free projections) and noise (from noisy projections) of the reconstructed images.

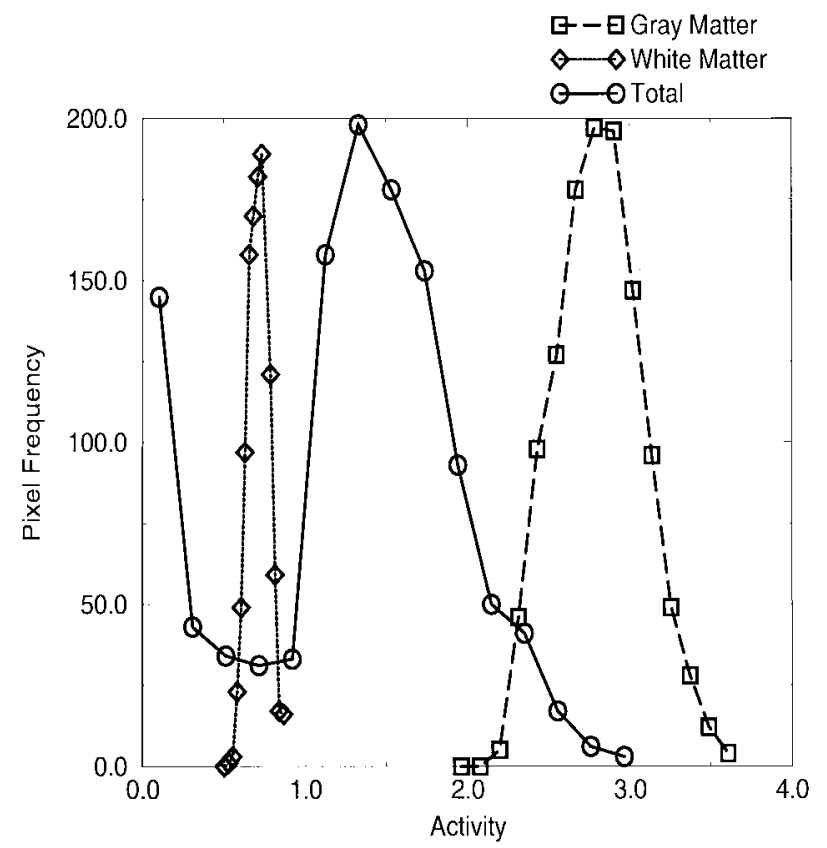

Fig. 2. Histogram of total activity, gray matter, and white matter activity levels in the simulation.

The rms bias is calculated as

$$
\text { rms bias }=\left[\frac{1}{N_{\text {pixels }}} \sum_{j}\left(\lambda_{j}^{\text {rec }}-\lambda_{j}^{\text {true }}\right)^{2}\right]^{(1 / 2)} \frac{100 \%}{\lambda_{\text {ave }}^{\text {true }}}
$$

where $\lambda_{j}^{\text {true }}$ are the true activities, $\lambda_{j}^{\text {rec }}$ are the reconstructed values, $N_{\text {pixels }}$ is the number of pixels in the brain, and $\lambda_{\text {ave }}^{\text {true }}$ is the average true activity throughout the brain. We estimate the bias from reconstructions of noise-free projections, since this method frees the bias estimates from the influence of noise in the reconstruction. Note that bias calculated in this manner is an approximate estimate, since the reconstruction algorithm is nonlinear. An alternate procedure is to obtain bias estimates from the mean reconstructed image of a large sample of noisy projection datasets. In this method, however, the estimated bias is not free of contributions due to noise.

The noise in the reconstructed images is estimated from the $\mathrm{SD}$ of pixel values across replicates

$$
\text { Noise }=\left[\frac{1}{10 N_{\text {pixels }}} \sum_{j, r}\left(\lambda_{j}^{r}-\lambda_{j}^{\text {ave }}\right)^{2}\right]^{(1 / 2)} \frac{100 \%}{\lambda_{\text {ave }}^{\text {true }}}
$$

where $r$ indexes the ten independent realizations and $\lambda_{j}^{\text {ave }}$ is the average pixel value across the replicates. Although ten replicates is insufficient to accurately estimate the noise for a single pixel, the noise estimate in (26) is based on the entire image, and is, thus, much more reliable.

For comparison, reconstructions using algorithms that directly estimate $\lambda_{j}$ without the tissue-type information were also performed. We obtained 1) the unconstrained EM-ML reconstruction, and 2) MAP estimations with a Gibbs smoothness constraint using the same form as the Gibbs term in the Smoothness-Gaussian prior (10), but applied to the total pixel activities $\lambda_{j}$. 
In all of these analyses, the assumptions of the simulation model were well matched to the form of the priors. We, therefore, also performed two error analyses: 1) including a localized region of high activity not identified in the anatomical information, and 2) introducing registration errors between the MR segmentation and the PET projection data. In the first case, we generated a true image (and the corresponding projection data) with a "hot spot" in a region of mostly GM of $\sim 1-\mathrm{cm}$ diameter where the GM activity was 1) $25 \%$, 2) $50 \%$, and 3) $100 \%$ higher than the average GM activity. To assess the bias in this case, we calculated the "Contrast Recovery" defined as

$$
\text { Contrast Recovery }=\frac{\left(\Lambda_{H}^{\text {rec }}-\Lambda^{\text {true }}\right)}{\left(\Lambda_{H}^{\text {true }}-\Lambda^{\text {true }}\right)} 100 \%
$$

where $\Lambda_{H}^{\text {rec }}$ is the mean over reconstructed activities in the region-of-interest (ROI) defining the hot spot, $\Lambda_{H}^{\text {true }}$ is the corresponding value in the true image with the hot spot, and $\Lambda^{\text {true }}$ is the original image value without the hot spot. In the second case, we generated true images shifted horizontally by 1,2 , and $4 \mathrm{~mm}$ so that the segmentation information used by the algorithm $\left(p_{j n}\right)$ was incorrect. Reconstructions were performed only for noise-free projections, since the objective here was to analyze the bias.

\section{RESULTS}

Fig. 3 shows reconstructed images for the Gaussian [Fig. 3(c)] and Smoothness-Gaussian priors [Fig. 3(d)], along with the EM [Fig. 3(a)] and EM with Gibbs prior [Fig. 3(b)] cases. The images shown are reconstructions for a single noisy projection realization. Visual assessment [comparing to the ideal image in Fig. 1(d)] suggests that the reconstructions using these priors display lower noise compared to conventional EM, and better resolution compared to EM with Gibbs prior. Note, of course, that neither the unconstrained EM-ML nor the EM with Gibbs prior incorporate the additional MR information. In addition, the quadratic form of the Gibbs prior used here does not have edge-preserving properties.

As expected, the reconstructed images are dependent on the value chosen for the constraint parameters. Fig. 4 shows this dependence for the Gaussian prior, for three different values of $K$ [1 (top row), 5 (middle row), and 20 (bottom row)]. Fig. 4(a)-(c) shows typical reconstructed images, which appear noisier as $K$ increases. This is confirmed in the SD images Fig. 4(g)-(i), which show that image noise increases rapidly with $K$. However, the bias in the reconstruction decreases as $K$ is increased, as can be seen by the bias images [Fig. 4(d)-(f)] (reconstructed image minus true image for reconstructions of noise-free projections). For $K=1$ (top row), the bias values in the image are large and show considerable structure, indicating that the prior is causing the tissue-type activity estimates to be "drawn to the mean." Thus, for pixels with high absolute activities, the bias is negative, and vice versa. As the strength of the prior is weakened (higher $K$ values, middle and lower rows in Fig. 4), this effect is diminished. Thus, reconstructions at different values of $K$ display a systematic tradeoff between noise and bias in the reconstructed images.

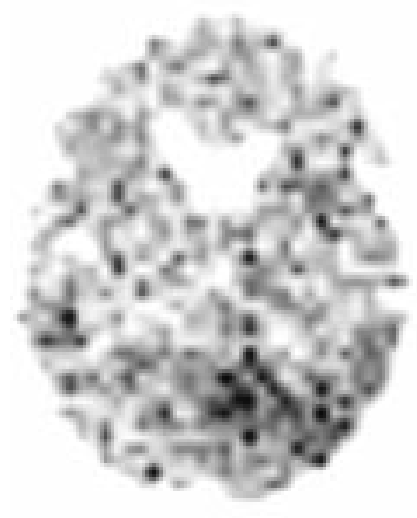

(a)

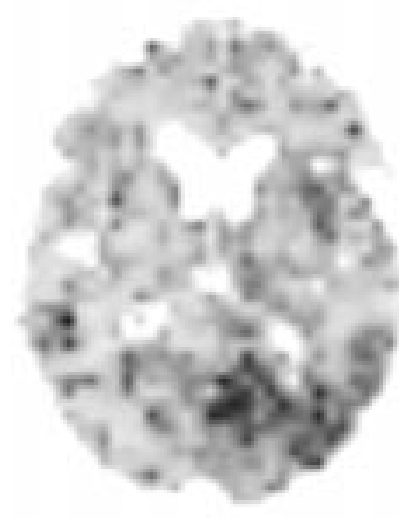

(c)

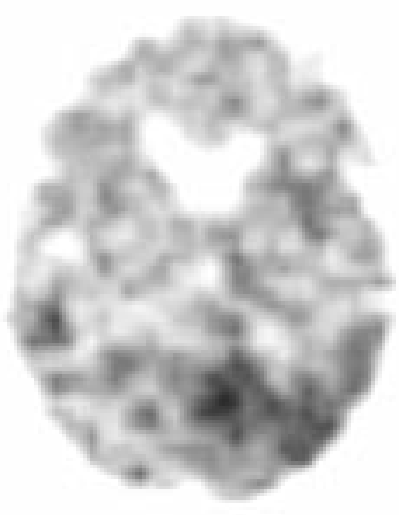

(b)

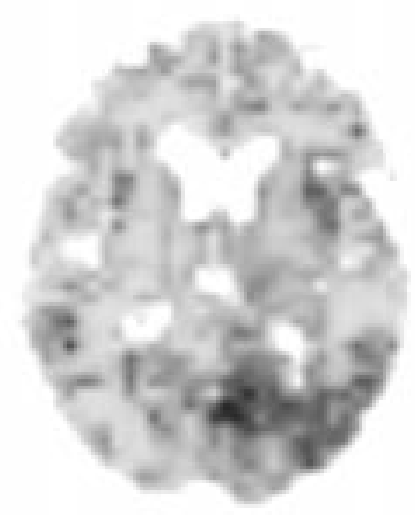

(d)
Fig. 3. Images from a single reconstruction with Poisson noise using (a) unconstrained EM algorithm, (b) EM algorithm with Gibbs prior $\left(K_{S}=5\right)$, (c) Gaussian prior $(K=5)$, and (d) Smoothness-Gaussian prior $\left(K=5, K_{S}=5\right.$ ). Images are displayed on a common scale of $0-250 \%$ of true mean activity.

The influence of the constraint parameter $K$ in forcing activity values to a narrower distribution is shown quantitatively in Fig. 5, where we plot binned error values (reconstructed activities minus true activities) against true activities from noise-free reconstructions. These data are generated by binning pixels according to their known true activities, considering only pixels which are predominantly GM $\left(p_{G M}>0.5\right)$. For $K=1$, pixels with lower true activity have reconstructed activities which are in excess of the true values, and for high true activity, the case is the reverse. This bias diminishes as $K$ increases.

Fig. 6 shows the full relationship of rms noise and bias values for all the algorithms. First, conventional EM (solid square) shows high noise as well as a large rms bias. The latter appears to be largely due to incomplete convergence. Examination of the rms bias values as a function of iterations (data not shown) shows that the EM bias is still changing, even at 1000 iterations, while the bias values for the Gaussian priors show only small changes after tens of iterations. This is not the case for ROI values, which show more rapid convergence than individual pixel values [2]. When a Gibbs smoothing prior is added to the conventional EM algorithm (open squares), as expected, noise decreases and bias increases. 


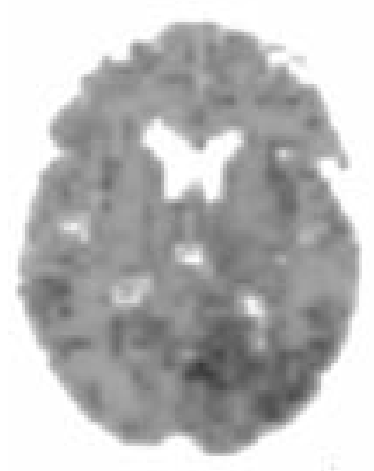

(a)

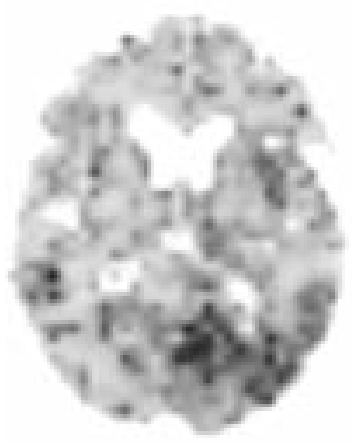

(b)

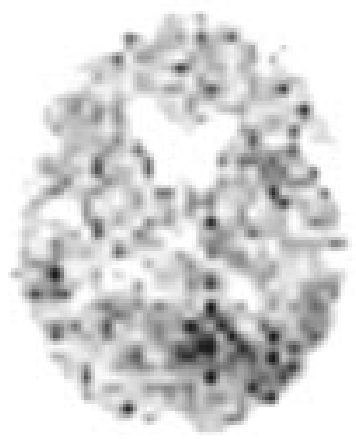

(c)

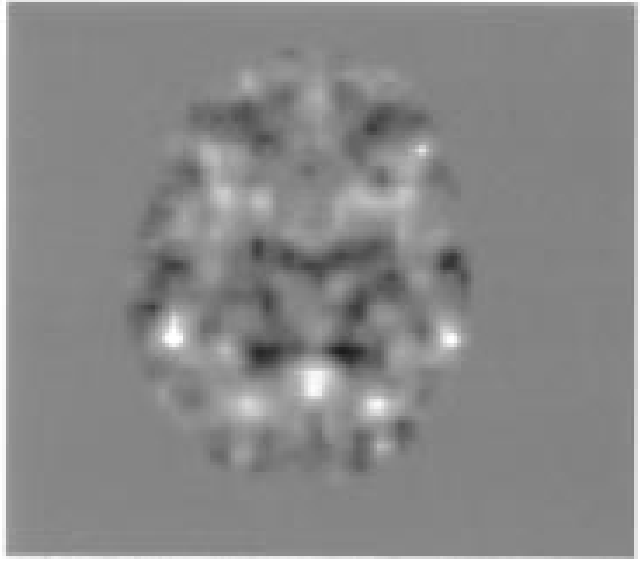

(d)

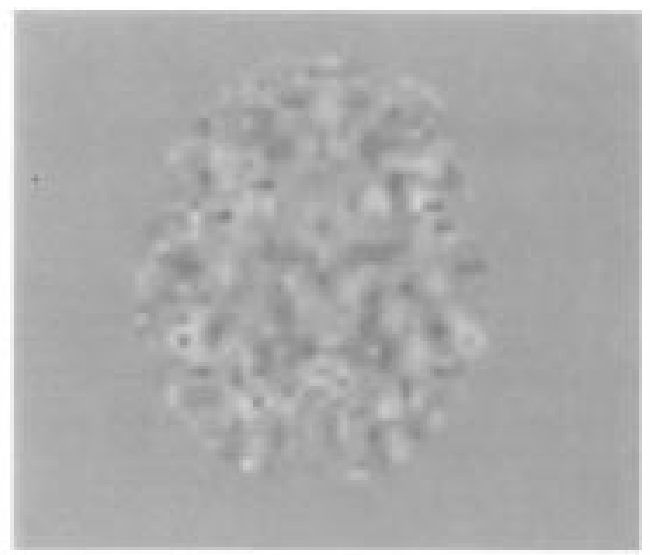

(e)

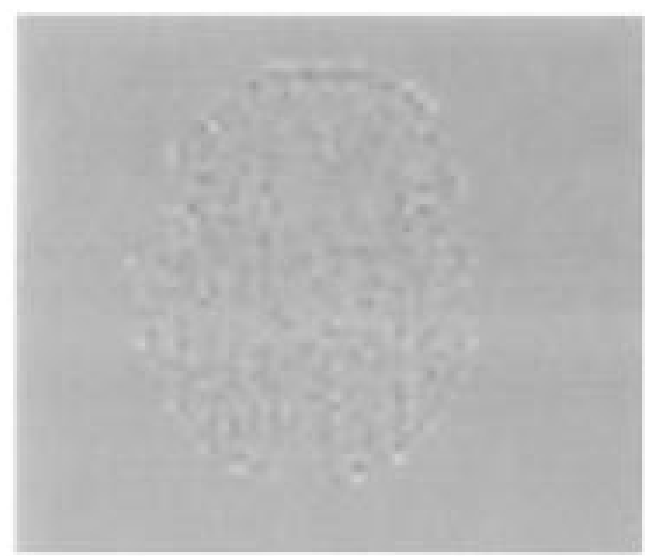

(f) (g)

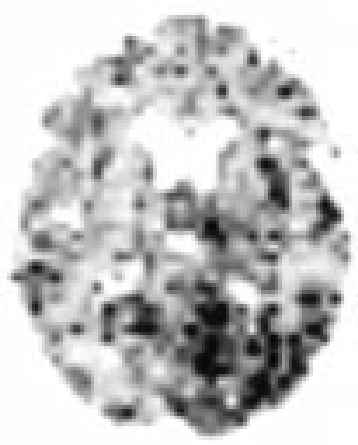

(h)

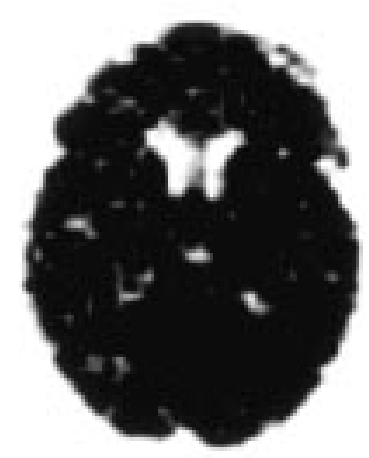

(i)

Fig. 4. (a)-(c) Sample reconstructed images, (d)-(f) bias images (reconstructed image from noise-free data-true image), and (g)-(i) SD images for the Gaussian prior with $K=1$ (top row), $K=5$ (middle row) and $K=20$ (bottom row). The display scale is $0-250 \%$ of true mean activity for (a)-(c), $-25 \%$ to $+25 \%$ for (d)-(f), and $0-25 \%$ for (g)-(i).

The introduction of the priors demonstrates substantial improvement in these images by moving the noise-bias curve toward the origin. The Gaussian prior (filled circles) shows an increase of noise and decrease of bias as $K$ increases. When the smoothness prior is added with a range of $K_{S}$ values for Gaussian constraint $K=5$ (open circles) and $K=20$ (open triangles), the noise-bias relationship is further improved. Note that the Smoothness-Gaussian cases asymptotically approach the bias and noise values of the corresponding Gaussian case as the smoothness constraint is relaxed ( $K_{S}$ increases).

It can be seen in Fig. 4 (compare SD images with reconstructed images) that the level of noise in pixels is roughly proportional to the activity. To see this more clearly, we show in Fig. 7 the dependence of noise on the pixel activities. The noise shown is normalized in each reconstruction to the global level of noise for the full image. For the EM case and for 


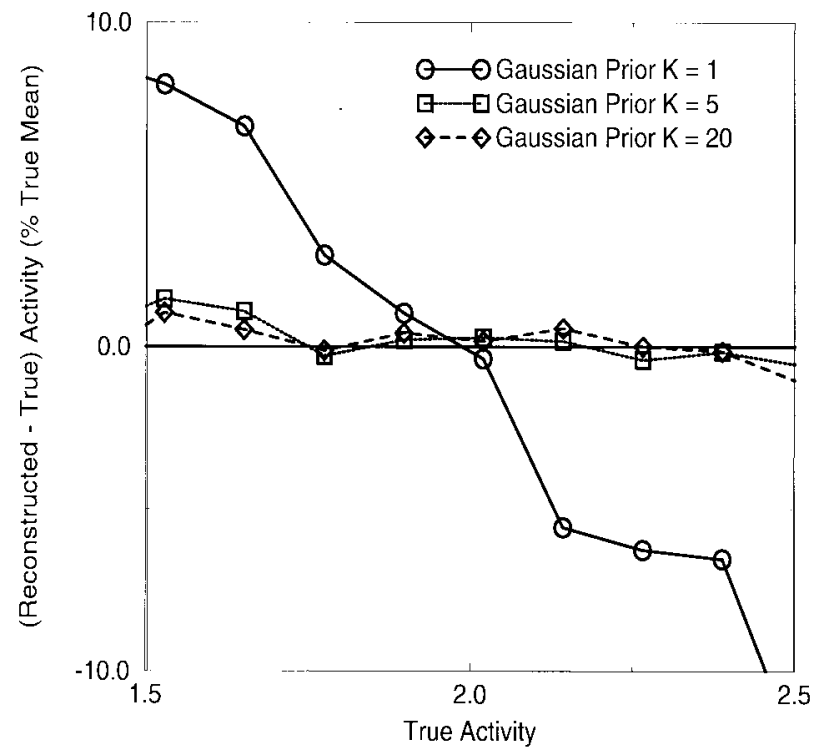

Fig. 5. Difference of reconstructed and true activities plotted against true activities for predominantly GM pixels $\left(p_{G M}>0.5\right)$ for Gaussian prior with $K=1,5$, and 20 .

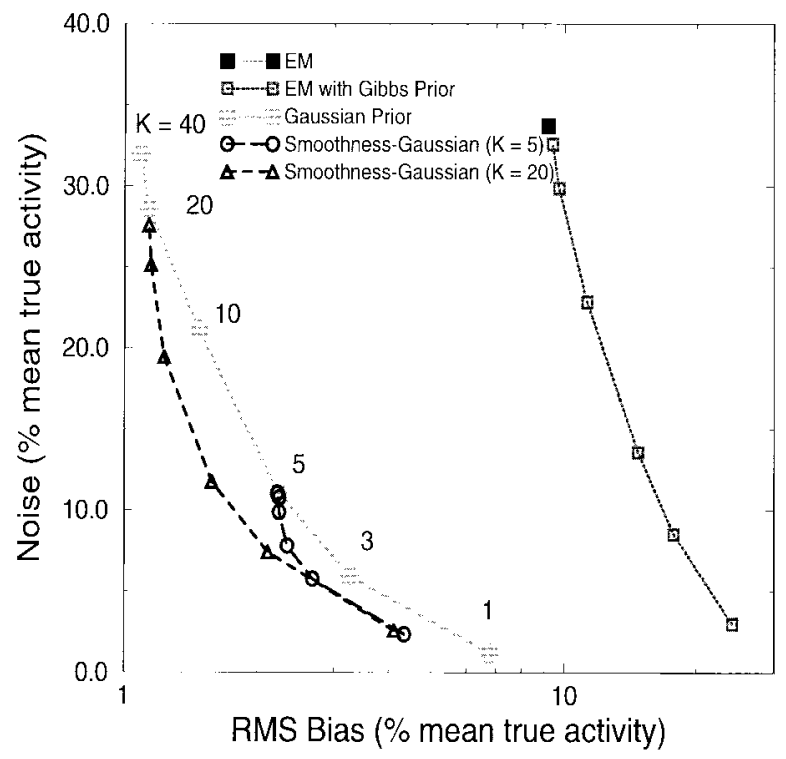

Fig. 6. RMS bias (25) versus noise (26) expressed as percentages of true mean activity, for the EM (filled square), EM with Gibbs (open squares), Gaussian prior (filled circles), and Smoothness-Gaussian prior [with $K=5$ (open circles) and $K=20$ (open triangles)] reconstructions. The values of the control parameter $K$ for the Gaussian prior are indicated next to the data points. For the Smoothness-Gaussian cases, the $K_{S}$ values range from 1-40 with smaller $K_{S}$ values showing smallest noise and largest bias.

a weak application of the prior $(K=20)$, the noise shows a "square-root" dependence. For a strong imposition of the Gaussian constraint, the noise is roughly linearly proportional to the activity. This behavior is consistent with the Poisson nature of the data model and the fact that in the Gaussian constraint, we choose the FWHM to be proportional to the activity value.

It is relevant to inquire, given the features of the reconstruction algorithm here, whether any significant dependence exists between noise in the reconstruction and the composition

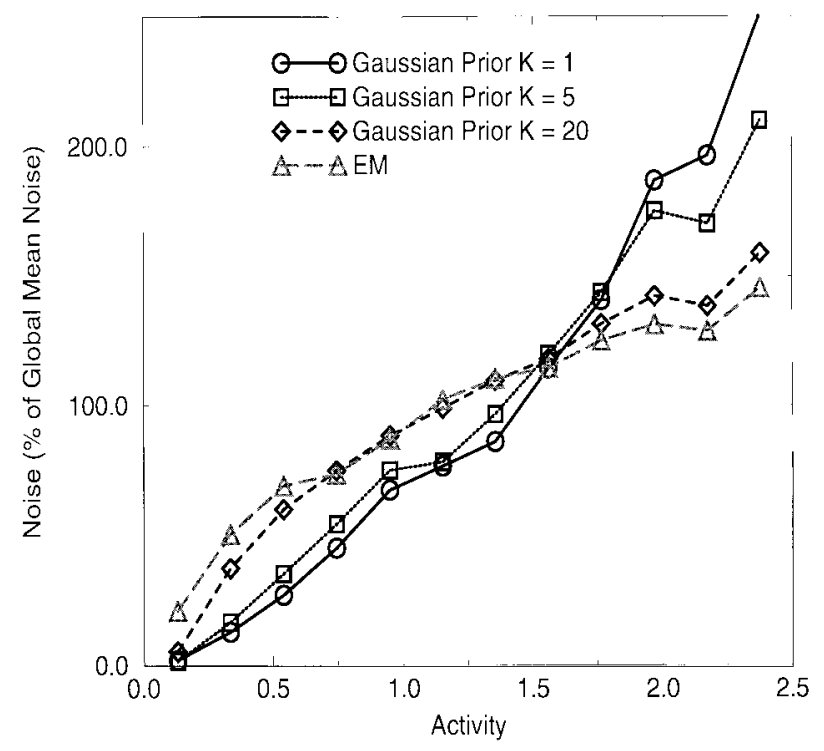

Fig. 7. Noise (normalized to a mean of 100\%) versus Activity for reconstructions with the Gaussian prior and the EM reconstruction. The shape of the curves changes as the control parameter $K$ is varied. The $K=20$ case has very similar characteristics to the conventional EM data.

of a given pixel. In other words, will estimates from pixels that are predominantly a single tissue type have the same characteristics as those from heterogeneous pixels? A standard way to characterize the heterogeneity is to calculate for each pixel the Shannon entropy, $S_{j}=-\Sigma_{n} p_{j n} \ln \left(p_{j n}\right)$. First, we removed the influence of pixel activity on noise (Fig. 7) with a polynomial fit. Then we tested whether there was any correlation between the residuals from that fit and the entropy values, but no detectable correlation was found (data not shown). Therefore, there was no evidence that "heterogeneous" pixels have higher noise than homogeneous pixels, although in this simulation the number of homogeneous pixels was small (Fig. 2).

While the initial performance results of these algorithms are promising, we recognize that the assumptions of the simulation are well matched to the form of the priors. Care should be taken in interpreting these results, since they are most likely better than what may be expected in practice. To assess their performance in more "real-world" situations, we considered two situations where deviations from the model could easily occur. The first is the presence of a local region with abnormally high activity. For true images containing a hot spot with 1) $25 \%$,2) $50 \%$, and 3) $100 \%$ increase in GM activity, Fig. 8 shows the contrast recovery as a function of $K$ for reconstructions with the Gaussian prior. For low values of $K$, there is a substantial underestimate of the true contrast due to the "flattening" influence of the prior. However, contrast recovery clearly improves with $K$, reaching $\sim 90 \%$ for $K=10$ for all levels of abnormal activity. Note that these values are comparable to the results for conventional EM which showed contrast recovery of $86 \%, 92 \%$, and $94 \%$ for the $25 \%, 50 \%$, and $100 \%$ hot spots, respectively. The contrast recovery for large $K$ values is actually better for the $100 \%$ hot spot compared to the $25 \%$ case. However, since contrast recovery is calculated as a percentage of true contrast, the 


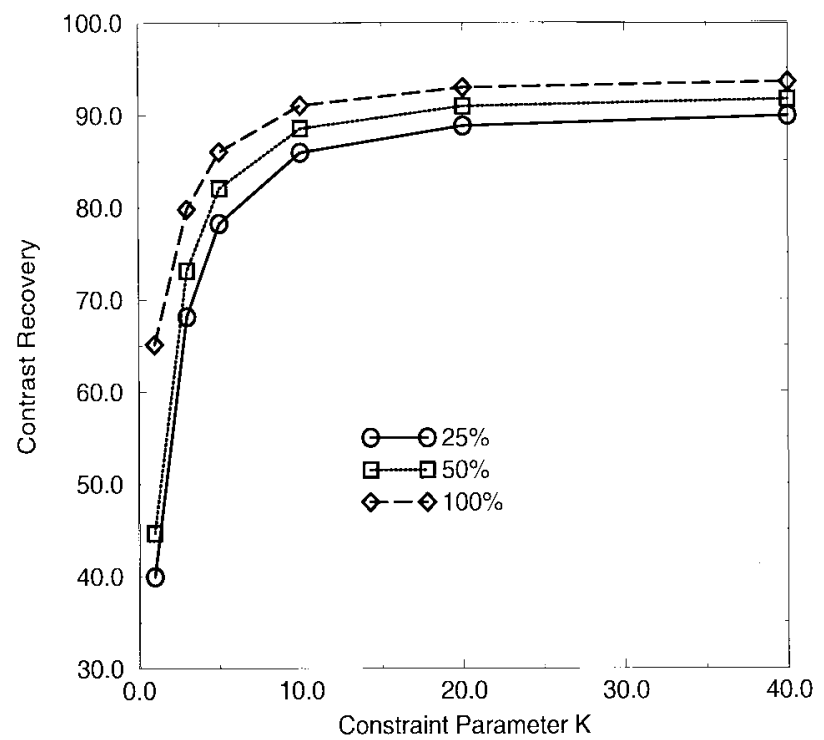

Fig. 8. Contrast recovery (27) as a function of constraint parameter $K$ for the Gaussian prior, for three different levels of local hot spot activity not included in the prior information. Data shown are from reconstructions of noise-free projections.

absolute magnitude of the bias for the $100 \%$ case is in fact the largest.

The second error analysis that we performed concerned the effect of registration errors. This situation was studied by shifting (to the right) the segmentation profile used in the reconstruction relative to the segmentation used in creating the true image. Fig. 9 shows the bias images in the case of a registration error of $2 \mathrm{~mm}$ for a range of values of $K$ in reconstructions with the Gaussian prior. The error in the reconstruction is substantial, increasing approximately linearly with registration error and diminishing with increasing $K$. The rms bias values for $K=1$ are $10.3 \%, 18.2 \%$, and $33.5 \%$ for shifts of 1,2 , and $4 \mathrm{~mm}$, respectively. For $K=$ 40 , these biases reduce to $5.4 \%, 11.8 \%$, and $22.5 \%$. Such sensitivity to registration errors has also been observed in another MR-based reconstruction method that uses a similar Gaussian prior [27]. An interesting feature to be noted is the asymmetry in these bias images for higher values of $K$. On the left edge of the image (where the true image contained significant activity from GM, while the segmentation used in the reconstruction specified that there is no GM in these pixels) the bias persists even as $K$ is increased. On the right edge (where the segmentation used in the reconstruction assumed the presence of GM, while in the true image, these pixels lie in CSF or outside the brain) the error diminishes as $K$ increases. This occurs because the reconstruction allows some variability within each tissue type which we have expressed as a percentage $(K \%)$ of the mean activity in each tissue. Therefore, on the right edge, it can accommodate the low activity levels in pixels misassigned to GM. However, on the left edge, the model will not permit such a high activity level in pixels misassigned to be CSF.

\section{DISCUSSION}

We have presented a Bayesian reconstruction algorithm for PET which utilizes segmented MR anatomical data to define

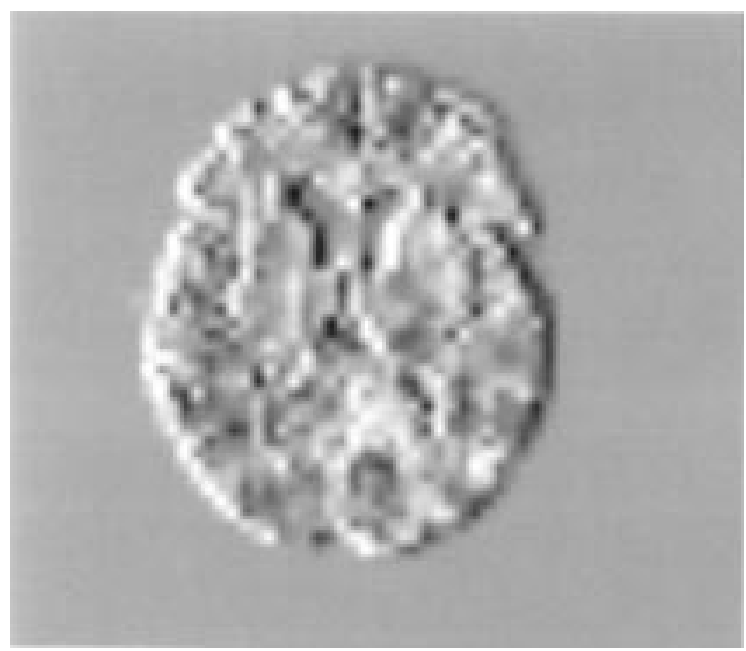

(a)

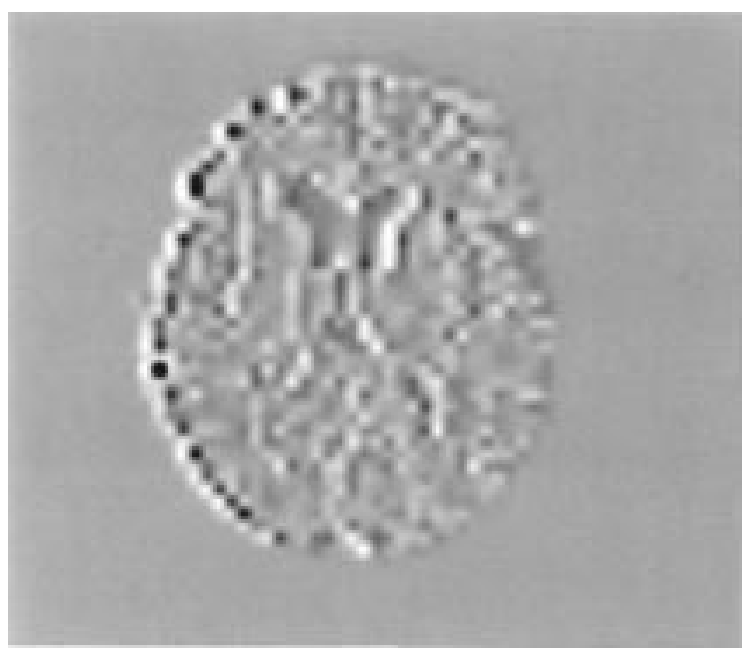

(b)

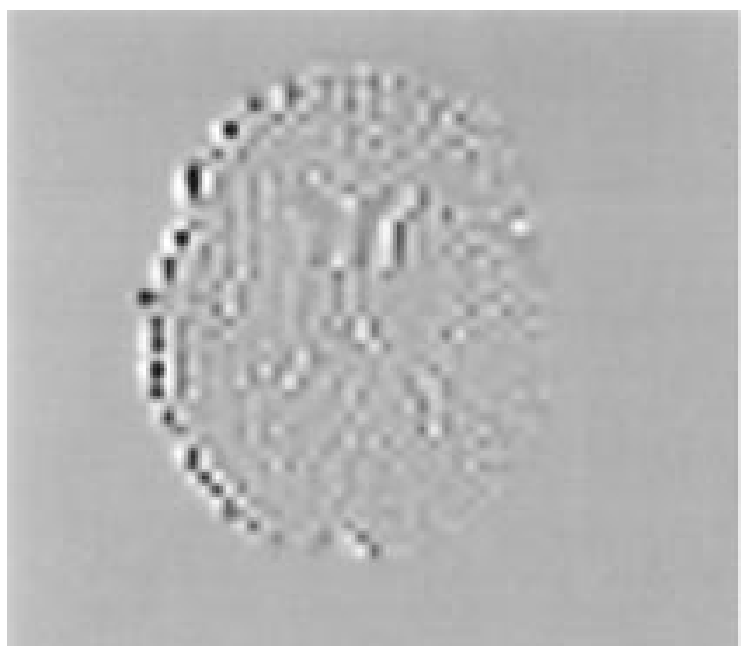

(c)

Fig. 9. Difference images between true and reconstructed images in the case of a horizontal registration error of $2 \mathrm{~mm}$. The reconstructions are performed with the Gaussian prior, with noise-free projections. (a) $K=1$. (b) $K=5$. (c) $K=20$. The display scale is $-50 \%$ to $+50 \%$ of true mean activity. 
a prior density for the image activities. The Gaussian prior imposes a constraint on the overall variability of activity levels for individual tissue types. The Smoothness-Gaussian prior adds assumptions about the smoothness of the activity values within each tissue type. The reconstructed images display reduced bias and noise compared to the EM reconstruction or the MAP estimation with a smoothness prior. For the priors we consider, a systematic tradeoff between noise and bias is observed as a function of the control parameter values. Violations of the assumptions of the prior, for example due to misregistration between the MR and PET data, can introduce large biases, particularly for the values of the control parameters that produce the greatest noise reduction.

As noted in Section III, we iteratively update values of the global and local mean activities $\bar{\lambda}_{n}$ and $\bar{\lambda}_{j n}$. Hence, reconstructed images discussed in Section $\mathrm{V}$ are not rigorous MAP estimates because the Bayesian parameters were not defined a priori. One motivation for this approach was to reduce the bias in the initial estimates of the global-mean activities $\bar{\lambda}_{n}$. In the simulations performed here, the initial estimates of mean GM and WM activity deviated from the true mean activities by $+4.7 \% \pm 0.5 \%$ and $-10.0 \% \pm 1.8 \%$, respectively. However, in reconstructions with the Gaussian prior $(K=5)$ where these mean values were updated iteratively, at 500 iterations, the bias in mean GM and WM activities was reduced to $+2.0 \% \pm 0.6 \%$ and $-4.9 \% \pm$ $2.6 \%$, respectively. The same bias reduction was found for the Smoothness-Gaussian prior $\left(K=5, K_{S}=5\right)$. This suggests that it is useful to iteratively update the global-mean activities. To determine the effect of increased bias in $\bar{\lambda}_{n}$ on the final image values, we performed reconstructions with the Gaussian prior, wherein we kept the global-mean activities $\bar{\lambda}_{n}$ fixed at their initial values. We found $5-10 \%$ increases in rms bias and $0.2-2 \%$ increases in noise with the fixed-parameter algorithm. For example, for $K=5$, the bias for fixed $\bar{\lambda}_{n}$ was $2.34 \%$ compared to $2.23 \%$ for the algorithm with iteratively updated values of $\bar{\lambda}_{n}$. For $K=20$, the bias for the fixed-parameter algorithm was $1.23 \%$ compared to $1.14 \%$. These observations suggest that there may be a small advantage to iteratively updating the global tissue-type activities, particularly if there are larger errors in the initial estimates of $\bar{\lambda}_{n}$ than those obtained here. However, with good initial estimates of global activity levels, the algorithm's performance does not crucially depend on iterative updating of the prior parameters.

A new feature in our work is the introduction and estimation of tissue-type activities $\lambda_{j n}$ in a reconstruction algorithm. In so doing, the number of unknowns has increased by a factor equal to the number of tissue types. For example, in our simulations with four tissue types, the number of variables $\lambda_{j n}$ to be estimated exceeds the number of projection values $Y_{i}$ by $33 \%$. Given that unconstrained EM estimation of the $\lambda_{j}$ values alone is already a slowly converging procedure, it is clearly not feasible to estimate the $\lambda_{j n}$ values in such a manner. However, the inclusion of priors in the reconstruction method adds the regularization necessary to make it possible to estimate them. This additional information provided by the decomposition of the pixel-wise activities $\lambda_{j}$ into contributions from individual tissue types $\lambda_{j n}$ is potentially useful, but further study is needed to validate this idea. In the results described, we have focused on the estimates of total activity rather than tissue-type activity levels to allow comparison with existing algorithms.

The appropriate number of tissue types to use in a given reconstruction problem will vary with the organ of interest and the radioisotope distribution. Here, we defined tissue type based on MR images and, therefore, the number of tissue types depends upon the segmentation algorithm. In the simulations performed here, this number is four (CSF, GM, WM, and other).

One of the goals of model-based iterative reconstruction is to provide radioactivity measurements that are free of the partial-volume effect [28] in order to permit accurate quantification in small structures. With the incorporation of an accurate projection model (including resolution effects), this can theoretically be achieved. Conventional EM-ML can produce unbiased estimates [2], but given the limited counting statistics of PET data, it suffers from a large increase in noise, as well as slow convergence at the pixel level. Multimodality Bayesian approaches, such as the algorithms presented here, have the potential to produce PET reconstructions without partial volume effects at reasonable noise levels. These techniques for ideal quantification could be very important for problems such as atrophy correction. Note that there are also post-reconstruction methods for removing the partial volume effect (e.g., [29]) which depend on accurate anatomical information. We expect that incorporating the anatomical information directly into the reconstruction would produce somewhat better results (smaller bias and/or lower noise), although this remains to be demonstrated.

To achieve the goal of very high-resolution functional images with moderate noise levels, we need accurate anatomical information. Here, we showed that misregistration of the anatomical and functional information can introduce serious errors. However, the accuracy of registration techniques is generally quite good (registration errors less than $2 \mathrm{~mm}$ ) [30] and, hence, this requirement is not expected to pose a significant practical problem. The effects of misregistration might be reduced by the inclusion of uncertainty in the registration in determining the tissue composition, $p_{j n}$, and the strength of the constraint applied via the prior. Alternatively, registration could be incorporated directly into the reconstruction process, in which case, a desirable procedure is to reconstruct all slices simultaneously. Simultaneous reconstruction of all slices is also likely to improve the estimation of the global activity $\bar{\lambda}_{n}$.

The effects of missegmentation must also be considered. In the error analysis data presented here, there was a moderate bias in the presence of localized regions with substantially different activity than predicted from the segmentation. The contrast recovery is seen to be roughly independent of the level of excess activity in the case of the Gaussian prior. This can be understood qualitatively from the fact that although the constraint strength in the model prior is an indication of the degree of variability in tissue-type activity, as seen from (18), the constraint parameter, in effect, determines the balance between the influence of the measured projections, and that of the global-mean value on the iterative update of $\lambda_{j n}$. 
In cases where missegmentation is of concern, our data on contrast recovery suggest that an appropriate choice of prior might be a Smoothness-Gaussian prior with a permissive global constraint (large $K$ ). In this way, contrast recovery in the missegmented region may be maintained while keeping the noise reduction advantages of the smoothness prior. Note, however, that the smoothing within tissue type produced by this prior will cause some loss of contrast if the ROI has been assigned to an inappropriate tissue type. It is also possible that a nonquadratic form for the global-mean constraint would produce less bias in cases of abnormal local activity.

In this study, we did not supply a proof of algorithm convergence or perform an evaluation of convergence or optimal iteration stopping criteria, but simply restricted ourselves to images obtained at 500 iterations. Since convergence rates can differ between the algorithms, and since we estimated bias and noise characteristics at the resolution of individual pixels (albeit averaged over the image), we can expect that the results may change somewhat, if other convergence criteria were used. Also, we have not searched for the optimum pixel size for the present algorithm. For unconstrained EM-ML, performance for ROI quantification does not improve with smaller pixel sizes [2]. It is possible that for the present algorithm, performance will improve for smaller pixels, although sensitivity to registration error may increase, convergence may be slower, and computation time will increase. Nevertheless, our principal interest was in the feasibility of utilizing anatomical prior data in this manner by the definition of new a posteriori likelihood functions, and not in the convergence of the particular iteration scheme used here. If these likelihood functions do in fact produce images with useful characteristics, then a rapidly converging algorithm should be developed.

A feature in our method, in common with other reconstruction methods using prior functions, is the presence of tunable parameters (constraint strength parameters $K$ and $K_{S}$ ) defining the priors. Such hyperparameters need to be chosen properly in order to obtain satisfactory image characteristics [31]-[33]. In the context of the tissue composition model, the meaning of the hyperparameter $K$ is related to identifiable physiological details, i.e., the percent variability of radioactivity levels within each tissue type. The appropriate choice of $K$ depends on the particular imaging task. For example, if absolute quantification (i.e., bias reduction) is important, then Fig. 6 suggests that a value of $K$ between 5 and 10 for the Gaussian prior might be an appropriate choice. Note, however, that the "true" image was constructed to have a variability of $\sim 20 \%$ of the mean activity level for each tissue type. Thus the quantitative relation between the optimum value of the hyperparameter $K$ and the inherent variability is not trivial; it may be expected, for example, that the best choice depends also on the number of counts present in the projection data.

\section{ACKNOWLEDGMENT}

The authors wish to thank Dr. J. VanMeter for the segmentation data. They would also like to acknowledge the helpful comments of Dr. M. Daube-Witherspoon, Dr. S. Bacharach, Dr. G. Weiss, and Dr. S. Pajevic. Finally, the comments of the manuscript reviewers are also greatly appreciated.

\section{REFERENCES}

[1] L. A. Shepp and Y. Vardi, "Maximum likelihood reconstruction for emission tomography," IEEE Trans. Med. Imag., vol. MI-1, pp. 113-122, 1982.

[2] R. E. Carson, Y. C. Yan, B. Chodkowski, T. K. Yap, and M. E. Daube-Witherspoon, "Precision and accuracy of regional radioactivity quantitation using the maximum-likelihood EM reconstruction algorithm,"IEEE Trans. Med. Imag., vol. 13, pp. 526-537, 1994.

[3] D. L. Snyder, M. I. Miller, L. J. Thomas, and D. G. Politte, "Noise and edge artifacts in maximum-likelihood reconstruction for emission tomography," IEEE Trans. Med. Imag., vol. MI-6, pp. 228-238, 1987.

[4] E. Levitan and G. T. Herman, "A maximum a posteriori probability expectation maximization algorithm for image reconstruction in emission tomography," IEEE Trans. Med. Imag., vol. MI-6, pp. 185-192, 1987.

[5] T. Hebert and R. Leahy, "A generalized EM algorithm for 3-D Bayesian reconstruction for Poisson data using Gibbs priors," IEEE Trans. Med. Imag., vol. 8, pp. 194-202, 1989.

[6] V. E. Johnson, W. H. Wong, X. Hu, and C. Chen, "Bayesian reconstruction of PET images using Gibbs priors," in Information Processing in Medical Imaging, D. A. Ortendahl and J. Llacer, Eds.. New York: Wiley-Liss, 1989, pp. 15-28.

[7] Z. Liang, R. Jaszczak, and K. Green, "On Bayesian image reconstruction from projection: uniform and nonuniform a priori source information," IEEE Trans. Med. Imag., vol. 8, pp. 227-235, 1989.

[8] P. J. Green, "Bayesian reconstructions from emission tomography data using a modified EM algorithm," IEEE Trans. Med. Imag., vol. 9, pp. 84-93, 1990.

[9] K. Lange, "Convergence of EM image reconstruction algorithms with Gibbs smoothing," IEEE Trans. Med. Imag., vol. 9, pp. 439-446, 1990.

[10] J. Nunez and J. Llacer, "A fast Bayesian reconstruction algorithm for emission tomography with entropy prior converging to feasible images," IEEE Trans. Med. Imag., vol. 9, pp. 159-171, 1990.

[11] D. S. Lalush and B. M. W. Tsui, "Simulation evaluation of Gibbs prior distributions for use in maximum a posteriori SPECT reconstructions," IEEE Trans. Med. Imag., vol. 11, pp. 267-275, 1992.

[12] S. J. Lee, A. Rangarajan, and G. R. Gindi, "Bayesian image reconstruction in SPECT using higher order mechanical models as priors," IEEE Trans. Med. Imag., vol. 14, pp. 669-680, 1995.

[13] S. Geman and D. Geman, "Stochastic relaxation, Gibbs distributions and the Bayesian restoration of images," IEEE Trans. Pattern Anal. Machine Intell., vol. PAMI-6, pp. 721-741, 1984.

[14] C. T. Chen, X. Ouyang, W. H. Wong, X. Hu, V. E. Johnson, C. E. Ordonez, and C. E. Metz, "Sensor fusion in image reconstruction," IEEE Trans. Nucl. Sci., vol. 38, pp. 687-692, 1991.

[15] V. E. Johnson, W. H. Wong, X. Hu, and C. T. Chen, "Image restoration using Gibbs priors: boundary modeling, treatment of blurring, and selection of hyperparameter," IEEE Trans. Pattern Anal. Machine Intell., vol. 13, pp. 413-425, 1991.

[16] R. Leahy and X. Yan, "Incorporation of anatomical MR data for improved functional imaging with PET," in Information Processing in Medical Imaging, A. C. F. Colchester and D. J. Hawkes, Eds.. New York: Springer-Verlag, 1991, pp. 105-120.

[17] G. Gindi, M. Lee, A. Rangarajan, and G. Zubal, "Bayesian reconstruction of functional images using anatomical information as priors," IEEE Trans. Med. Imag., vol. 12, pp. 670-680, 1993.

[18] B. A. Ardekani, M. Braun, B. F. Hutton, "Improved quantification with the use of anatomical information in PET image reconstruction," in Quantification of Brain Function. Tracer Kinetics and Image Analysis in Brain PET, K. Uemura, N. A. Lassen, T. Jones, and I. Kanno, Eds. Amsterdam: Elsevier Science Publishers B.V., 1993, pp. 351-359.

[19] X. Ouyang, W. H. Wong, V. E. Johnson, X. Hu, and C. T. Chen, "Incorporation of correlated structural images in PET image reconstruction," IEEE Trans. Med. Imag., vol. 13, pp. 627-640, 1994.

[20] Y. Zhang, J. A. Fessler, N. H. Clinthorne, W. L. Rogers, "Joint estimation for incorporating MRI anatomic images into SPECT reconstruction," in Conf. Rec. IEEE Nuclear Science Symp. and Medical Imaging Conf., Norfolk, VA, 1994, pp. 1256-1260.

[21] J. E. Bowsher, V. E. Johnson, T. G. Turkington, R. J. Jaszczak, C. E. Floyd., R. E. Coleman, "Bayesian reconstruction and use of anatomical a priori information for emission tomography," IEEE Trans. Med. Imag., vol. 15, pp. 673-686, 1996.

[22] H. S. Choi, D. R. Haynor, Y. Kim, "Partial volume tissue classification of multichannel magnetic resonance images-a mixel model," IEEE Trans. Med. Imag., vol. 10, pp. 395-407, 1991.

[23] M. A. Tanner, Tools for Statistical Inference. Berlin, Germany: Springer-Verlag, 1993 
[24] J. W. VanMeter and P. A. Sandon, "Identification of cortex in magnetic resonance images," SPIE: Biomedical Image Processing and 3-D Microscopy, vol. 1660, 1992.

[25] R. P. Woods, J. C. Mazziotta, and S. R. Cherry, "MRI-PET registration with automated algorithm," J Comput. Assist. Tomogr., vol. 17, pp. 536-546, 1993.

[26] L. Sokoloff, M. Reivich, C. Kennedy, M. H. Des Rosiers, C. S. Patlak, K. D. Pettigrew, O. Sakurada, and M. Shinohara, "The $\left[{ }^{14} \mathrm{C}\right]$ deoxyglucose method for the measurement of local cerebral glucose utilization: Theory, procedure, and normal values in the conscious and anesthetized albino rat," J. Neurochem., vol. 28, pp. 897-916, 1977.

[27] B. Lipinski, H. Herzog, E. Rota Kops, W. Oberschelp, and H. W. MüllerGärtner, "MR guided PET reconstruction and problems with anatomical misinformation," NeuroImage, 2:S31, 1995.

[28] E. J. Hoffman, S.-C. Huang, and M. E. Phelps, "Quantitation in positron emission computed tomography: 1. Effect of object size," J. Comput. Assist. Tomogr., vol. 3, pp. 299-308, 1979.

[29] H. W. Müller-Gärtner, J. M. Links, J. L. Prince, R. N. Bryan, E. McVeigh, J. P. Leal, C. Davatzikos, and J. J. Frost, "Measurement of radiotracer concentration in brain gray matter using positron emission tomography: MRI-based correction for partial volume effects," J. Cereb. Blood Flow Metab., vol. 12, pp. 571-583, 1992.

[30] S. C. Strother, J. R. Anderson, X. L. Xu, J. S. Liow, D. C. Bonar, and D. A. Rottenberg, "Quantitative comparisons of image registration techniques based on high-resolution MRI of the brain," J. Comput. Assist. Tomogr., vol. 18, pp. 954-962, 1994.

[31] Z. Zhou, R. M. Leahy, and E. U. Mumcuoglu. "A comparative study of the effects of using anatomical priors in PET reconstruction" in Conf. Rec. IEEE Nuclear Science Symp. Medical Imaging Conf., 1993, pp. 1749-1753.

[32] S. J. Lee, G. R. Gindi, I. G. Zubal, and A. Rangarajan, "Using groundtruth data to design priors in Bayesian SPECT reconstruction" in Information Processing in Medical Imaging, Y. Bizais, C. Barillot, and R. D. Paola, Eds. Norwell, MA: Kluwer, 1995, pp. 27-38.

[33] Z. Zhou, R. M. Leahy, and E. U. Mumcuoglu, "Maximum likelihood hyperparameter estimation for Gibbs priors with applications to PET," in Information Processing in Medical Imaging, Y. Bizais, C. Barillot, and R. D. Paola, Eds. Norwell, MA: Kluwer, 1995, pp. 39-51. 